\section{A Practical Approach to Electrode-Skin Impedance Unbalance Measurement}

Enrique M. Spinelli*, Miguel A. Mayosky, and Ramon Pallás-Areny

\begin{abstract}
Unbalance between electrode-skin impedances is a major problem in biopotential recordings, leading to increased power-line interference. This paper proposes a simple, direct method to measure that unbalance at power-line frequency $(50-60 \mathrm{~Hz})$, thus allowing the determination of actual recording conditions for biopotential amplifiers. The method is useful in research, amplifier testing, electrode design and teaching purposes. It has been experimentally validated by using both phantom impedances and real electrode-skin impedances.
\end{abstract}

Index Terms-Biopotential amplifier, electrode-skin impedance, powerline interference.

\section{INTRODUCTION}

Power lines are a major source of electromagnetic interference (EMI) in biopotential measurements. Power line interference affects biopotential recording in many ways, as reported elsewhere [1]-[3]. Capacitive and inductive coupling between power lines and electrode leads can be reduced by carefully shielding and twisting those leads, but the displacement current ip coupled to the patient (see Fig. 1) yields a common mode voltage, which is higher in two-electrode systems than in systems using a third (ground) electrode. Because of unbalances between electrode impedances and/or common mode input impedances, that patient common mode voltage yields a differential mode voltage at the amplifier input, which is consequently amplified. That conversion from common to differential mode is also called "potential divider effect" and yields an input-referenced differential-mode interference voltage $V_{\text {D.EMI }}$ whose value is [1], [2]

$$
V_{\mathrm{D} . \mathrm{EMI}}=V_{\mathrm{CM}} \frac{Z_{\mathrm{E}}}{Z_{\mathrm{C}}}\left(\frac{\Delta Z_{\mathrm{E}}}{Z_{\mathrm{E}}}+\frac{\Delta Z_{\mathrm{C}}}{Z_{\mathrm{C}}}\right)
$$

where $V_{\mathrm{CM}}$ is the patient common mode voltage, $Z_{\mathrm{C}}$ is the average common-mode input impedance, $\Delta Z_{C}$ is the unbalance in that impedance, $Z_{\mathrm{E}}$ is the average electrode-skin impedance and $\Delta \mathrm{Z}_{\mathrm{E}}$ is the electrode-skin unbalance

$$
Z_{\mathrm{E}}=\frac{Z_{\mathrm{E} 1}+Z_{\mathrm{E} 1}}{2} ; \quad \Delta Z_{\mathrm{E}}=Z_{\mathrm{E} 1}-Z_{\mathrm{E} 1}
$$

Manuscript received December 22, 2004; revised January 22, 2005. This work was supported in part by the Consejo de Investigaciones Científicas y Técnicas (CONICET) under Grant PIP-5551. Asterisk indicates corresponding author.

*E. M. Spinelli is with the Laboratorio de Electrónica Industrial Control e Instrumentación, Facultad de Ingeniería, Universidad Nacional de La Plata (UNLP) and Consejo Nacional de Investigaciones Científicas y Técnicas CC 91, 1900 La Plata, Argentina (e-mail: spinelli@ieee.org).

M. A. Mayosky is with LEICI and Comisión de Investigaciones Científicas de la Provincia de Buenos Aires. CC 91, 1900 La Plata, Argentina (e-mail: mayosky@ieee.org).

R. Palls-Areny is with Instrumentation, Sensors and Interfaces Group, Castelldefels School of Technology (EPSC), Avda del Canal Olímpic S/N, Edifici C4, 08860 Castelldefels (Barcelona), Spain (e-mail: ramon.pallas@upc. edu).

Digital Object Identifier 10.1109/TBME.2006.875714

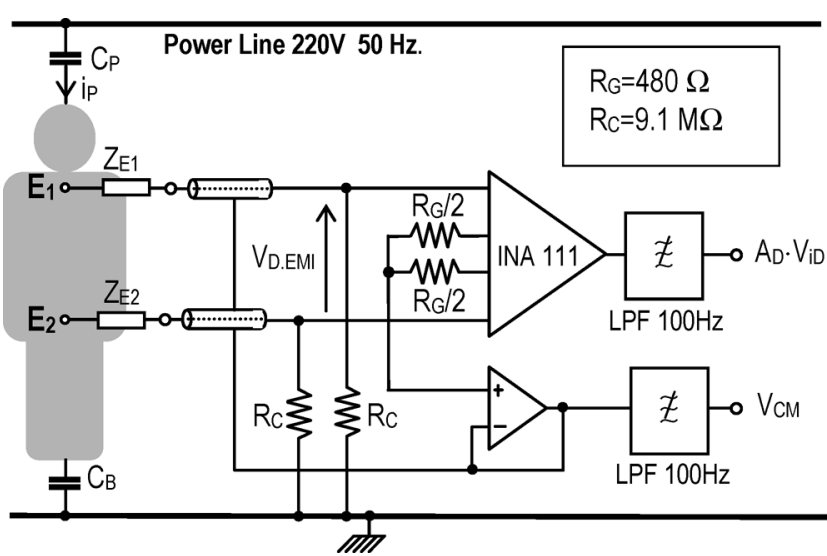

Fig. 1. System proposed to measure electrode-skin impedance unbalance.

Balanced common mode input impedances are not difficult to achieve by a careful amplifier design, but electrode impedances depend on skin preparation and site placement, so that their value is very difficult to control. As a result, the dominant interference voltage in (1) is

$$
V_{\mathrm{D} . \mathrm{EMI}}=V_{\mathrm{CM}} \frac{\Delta Z_{\mathrm{E}}}{Z_{\mathrm{C}}}
$$

which shows the importance of small electrode impedance unbalances to reduce interference. For amplifier testing, if we first determine electrode unbalance, the resulting interference will depend only on the amplifier [4]. On the other hand, in electrode design, if we know the common-mode input impedance of the amplifier, the interference will be a result of electrode impedance unbalance.

In order to check whether measurement conditions are acceptable or not (i.e. if the electrode-skin contact is good), many commercial systems measure individual electrode impedances ("electrode test" feature). However, that measurement is performed at frequencies other than power line frequency (usually $10 \mathrm{~Hz}$ ) and provides only qualitative information, which is not enough to verify good EMI measurement conditions because, according to (3), the interference does not depend on individual electrode impedances but on electrode unbalance at power-line frequency. Electrode unbalance measurements are required, for example, to test a new biopotential amplifier design under specific unbalance conditions [4], in high-resolution recordings (to minimize interference), and in electrode design (to test possible effects from different gel composition, for example). These measurements are also very instructive for students in laboratory experiments.

Standard impedance measurements are performed by injecting a current and sensing the potential difference across the electrode-skin interface. This implies that two series electrode-skin impedances are measured. An alternative "three-lead" technique can be used instead [5], which can measure single-electrode impedances. However, to avoid interaction with power-line interference, these measurements are not performed at power-line frequency. An ingenious measurement method working at power line frequency uses the power line itself to generate the current injected [6]. This method allows the measurement of single electrode impedances at 50-60 Hz and can be adapted for electrode unbalance measurements. However, determining electrode unbalance would imply two single electrode impedance measurements (each with real and imaginary parts) and then calculate their difference. This paper 
improves the technique described in [6] to directly determine electrode-skin impedance unbalance at power line frequency $(50-60 \mathrm{~Hz})$. The method does not require an ad-hoc circuit and any biopotential amplifier can be adapted to perform the measurement.

\section{MEASUREMENT METHOD} $\Delta Z_{\mathrm{E}}$

The proposed method starts from (3) by solving for the modulus of

$$
\left|\Delta Z_{E}\right|=\frac{\left|V_{\text {D.EMI }}\right|\left|Z_{\mathrm{C}}\right|}{\left|V_{\mathrm{CM}}\right|}
$$

If $Z_{\mathrm{C}}$ is known, measuring $\left|V_{\mathrm{D} . \mathrm{EMI}}\right|$ and $\left|V_{\mathrm{CM}}\right|$ yields $\left|\Delta Z_{\mathrm{E}}\right|$. The phase of $\Delta Z_{\mathrm{E}}$ could also be determined, indicating which electrode has the higher electrode-skin impedance, but this is not usually of much interest.

Fig. 1 shows a circuit to implement the proposed measurement method. The external resistors $R_{\mathrm{C}}$ ensure a well-known common mode input impedance. Their value must be high enough for the linear approximation implied in (1) to be acceptable, but small enough to ensure the potential divider effect predominate over the direct differential mode interference in Fig. 1 (power line voltage drop across the tissue between E1 and E2). A value of $R_{\mathrm{C}}$ of around $10 \mathrm{M} \Omega$ is a good compromise and limits patient fault currents to less than $50 \mu \mathrm{A}$. Two resistors $R_{\mathrm{C}}=9.1 \mathrm{M} \Omega$ ( $1 \%$ tolerance) were used. Obviously, if the amplifier has a known input resistance whose value is close to $10 \mathrm{M} \Omega$, there is no need to add external resistors $R_{\mathrm{C}}$. To apply the method to three-electrode amplifiers, the third (right-leg) electrode must be disconnected. The differential mode voltage $V_{\text {D.EMI }}$ is the input voltage of the instrumentation amplifier and the common mode voltage $V_{\mathrm{CM}}$ is obtained by splitting the gain-setting resistor $R_{\mathrm{G}}$ used in a three-op amp instrumentation amplifier and measuring the mid voltage. The output low pass filters reduce noise bandwidth by attenuating power line harmonics higher than 50 or $60 \mathrm{~Hz}$. Input protections (i.e. defibrillator protections, pacemaker pulse rejection circuits) do not affect the electrode unbalance measurement. Electrode leads were shielded and twisted to avoid interference effects not included in the derivation of (3).

The circuit in Fig. 1 applies to nonisolated systems, which are common in biopotential amplifier development, but can also be applied to isolated amplifiers, which are mandatory for measurements on patients. However, these yield a lower $V_{\mathrm{CM}}$ voltage (a few millivolts compared to up to $1 \mathrm{~V}$ for nonisolated systems), because only a fraction of the patient potential produces a common mode voltage [2]; most of the patient voltage drops across the impedance between the isolated ground and earth ground. For isolated amplifiers, is necessary to amplify $V_{\mathrm{CM}}$ and the voltages in (4) must be measured by using systems referred to the floating (signal) ground.

\section{EXPERIMENTAL RESULTS}

The proposed method has been validated by running several experiments using known electrode impedance unbalances (phantom), and real electrode impedances.

\section{A. Measurement of Known Impedance Unbalances}

This test was performed by placing one electrode on the patient and adding a resistor, simulating an electrode impedance unbalance, as shown in Fig. 2.

Differential $\left(V_{\mathrm{D} . \mathrm{EMI}}\right)$ and common mode $\left(V_{\mathrm{CM}}\right)$ potentials were measured using for $\Delta Z_{\mathrm{E}}$ metal film resistors (1\% tolerance) of $10 \mathrm{k} \Omega$ and $20 \mathrm{k} \Omega$. To verify the relationship given by (3) for each $\Delta \mathrm{Z}_{\mathrm{E}}$, different values for $V_{\mathrm{CM}}$ were obtained by modifying the patient cou-

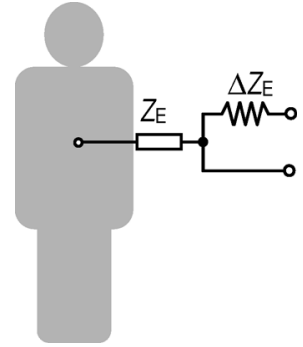

Fig. 2. Arrangement used to test the measurement method with known impedance unbalances.

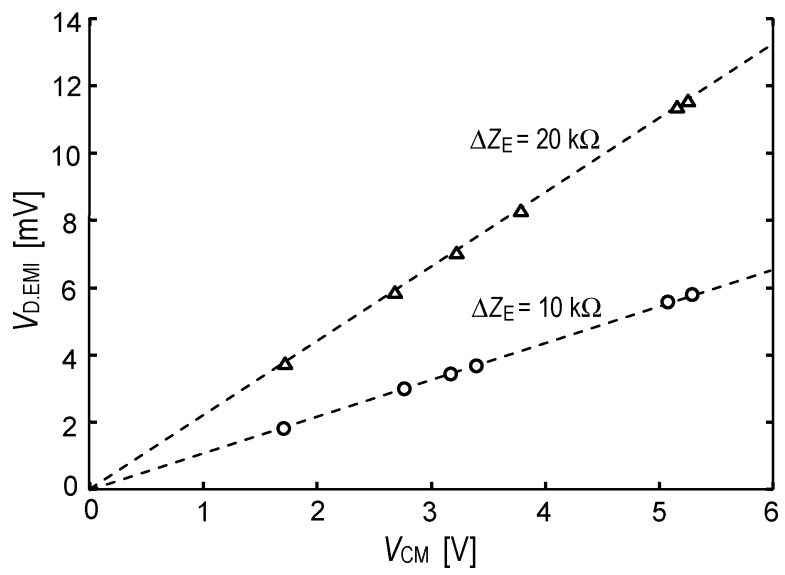

Fig. 3. Relationship between common mode and differential mode interference voltage for $\Delta \mathrm{Z}_{\mathrm{E}}=10 \mathrm{k} \Omega$ and $\Delta \mathrm{Z}_{\mathrm{E}}=20 \mathrm{k} \Omega$. Experimental data are indicated with markers and the theoretical curve in dashed line.

pling capacitance $C_{\mathrm{P}}$ in Fig. 1. This was accomplished, for example, by touching the subject, asking him to touch a large table, or by placing him close to an insulated power line cord.

The experimental results are plotted in Fig. 3. Experimental data points closely follow a straight line, as predicted by (3). The standard deviation between samples was small, so that a single measurement yields a good estimate of the electrode impedance unbalance.

\section{B. Measurement of Electrode-Skin Unbalances}

To measure actual skin-electrode impedances, two plate electrodes $\left(12 \mathrm{~cm}^{2}\right.$ area) were placed on the right and left inner arms of the patient (ECG lead I). The results are in Fig. 4 (circles). In a second experiment, the electrode impedance unbalance between a plate electrode (right arm) and a cup electrode (left arm) were measured, which should result in unbalances significantly larger than those obtained for two similar electrodes placed on the same locations. The experimental data are also shown in Fig. 4 (triangles). All the measurements were made 15 min after electrode application.

The results in Fig. 4 follow a straight line as predicted by (3). Nonsurprisingly, electrode unbalance is larger for dissimilar electrodes. The standard deviation of the unbalance was about $1 \%$. $V_{\mathrm{D} \text {.EMI }}$ and $V_{\mathrm{CM}}$ were both measured by a digital oscilloscope (Tektronix TDS3012) working in "averaging" mode triggered by "line," which "blurred" the ECG signal superimposed on $V_{\mathrm{D}}$.EMI. When $C_{\mathrm{P}}$ is large enough (i.e. when touching the subject), the interference signals can be measured with a standard digital multimeter.

\section{CONCLUSION}

A method for the direct measurement of electrode-skin unbalances at $50-60 \mathrm{~Hz}$ has been presented. It relies on the model described by (3) 


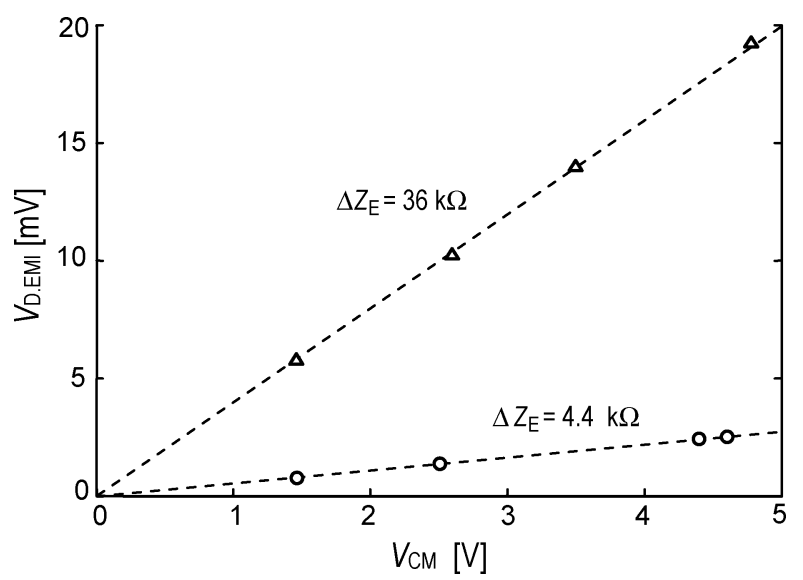

Fig. 4. Experimental data for the impedance unbalance between two plate (12 $\mathrm{cm}^{2}$ ) electrodes (circles) and between a plate electrode and a cup electrode (triangles).

an on a simple circuit that can be applied to most standard biopotential amplifiers. The method was validated with known unbalances (resistors) and also with real electrode-skin impedances. The results showed a very low dispersion (standard deviation of around 1\%). The method is simple to implement and can be used in amplifier and electrode research and also for teaching purposes.

\section{REFERENCES}

[1] J. C. Huhta and J. G. Webster, "60-Hz interference in electrocardiography," IEEE Trans. Biomed. Eng., vol. BME-20, pp. 91-101, Mar. 1973.

[2] A. C. Metting Van Rijn, A. Peper, and C. A. Grimbergen, "High-quality recording of bioelectric events: part 1 interference reduction, theory and practice," Med. Biol. Eng. Comput., vol. 28, pp. 389-397, Sep. 1990.

[3] M. Fernandez Chimeno and R. Pallas-Areny, "A comprehensive model for power line interference in biopotential measurements," IEEE Trans. Instrum. Meas., vol. 49, p. 53-540, Jun. 2000.

[4] E. M. Spinelli and M. A. Mayosky, "Two-electrode biopotential measurements: power line interference analysis," IEEE Trans. Biomed. Eng., vol. 52, no. 8, pp. 1436-1442, Aug. 2005.

[5] E. T. McAdams and J. Jossinet, "The importance of the electrode skin impedance in high resolution electrocardiography," Automedica, vol. 13, pp. 187-208, 1990.

[6] C. A. Grimbergen, A. C. Metting Van Rijn, and A. Peper, "A method for the measurement of the properties of individual electrode-skin interfaces and the implications of the electrode properties for preamplifier design," in Proc. IEEE 14th Annu. Int. Conf. Engineering in Medicine and Biology Soc., 1992, vol. 6, pp. 2382-2383. 